\title{
The Design and Implementation of the Micro Laser Engraving Machine Based on STM32
}

\author{
Zhenhua Wang ${ }^{1, a^{*}}$, Linna $\mathrm{Xu}^{2, \mathrm{~b}}$, Xianzheng $\mathrm{Su}^{3, \mathrm{c}}$ \\ ${ }^{1}$ College of electrical and information, Dalian Jiao tong University, China \\ ${ }^{2}$ College of electrical and information, Dalian Jiao tong University, China \\ ${ }^{3}$ College of electrical and information, Dalian Jiao tong University, China \\ a1126705914@qq.com, b467191255@qq.com, '993192801@qq.com
}

Keywords: Interpolation algorithm of numerical control; Laser engraving; Analysis of G code; The single-chip computer (MCU).

\begin{abstract}
Using STM32F103RBT6 as the core controller, using A3967 stepper motor drive circuit board for motion control of the stepper motor, with low power laser head, the mechanical part adopts Longmen milling machine structure, a two-dimensional axis screw slide, the design and implementation of a small laser engraving machine. The control system of the laser engraving machine can read $\mathrm{G}$ code data from the computer serial port or SD card, analyze G code through the single-chip computer (MCU) to carry out the interpolation calculating and control the motion of the stepper motor movement, achieving online and offline laser engraving function. The system implements the processing properties that a conventional laser engraving machine has, by a low cost, high precision and strong stability. The design contents include the mechanical structure, electrical hardware, as well as the MCU programming, this paper presents the detailed realization of the small laser engraving machine.
\end{abstract}

\section{Introduction}

As the name suggests, laser processing is the use of laser beam penetration on the surface of the object, including laser engraving, laser cutting, laser drilling, etc. According to the use of different occasions, you can choose different powers of laser head, the application of laser cutting, the general use of high-power laser head, the object surface carving, the general use of low-power laser head. The laser processing process is relatively complex, including material selection, surface treatment, punching, marking, scribing, with the progress of technology, the application of laser processing technology is more and more widely, has become one of the key technologies in the development of new industries, the transformation of traditional manufacturing industry[1]. For now, laser processing is widely used in medical equipment, metal processing, machinery manufacturing, metallurgy, petroleum processing, art processing, high-end packaging, personalized gift design and other fields. Through consulting relevant literature, the application of laser processing is more and more big.

Based on the above introduction, the design of the small laser engraving machine mainly includes the design of the lower computer, the software design of the host computer, and the design of the mechanical modeling and the writing of the control program. This design is aimed to build a a program framework for an economical and practical-type laser engraving machine[2], taking feasibility, accuracy, stability, innovation and expansibility into consideration, it shall possess all aspects of software \& hardware conditions of a complete system for the later flexible updating and upgrading in a wide range of applications. Through further analysis, difficult design issues including data transmission and storage, interpolation algorithm and $\mathrm{G}$ code parser design, in order to adapt to different situations, task set power regulator, can adjust the laser head power, so the research has great economic and social benefits [3]. 


\section{The Overall Design of the System}

The Control Structural Design of the System. The main purpose of the project is to design and implement a small laser engraving machine, can be used to process paper, leather, wood and other items, whose control structure is similar to that of the master-slave mode, but differs from it, in terms of behavior of online processing mode, the designed system belongs to the master-slave mode, because this model must rely on the mainframe computer to transfer processing data to the slave computer. In the offline processing mode of the system, the system can read processing data from SD card; the system itself is equivalent to a mainframe computer, so the subordination relation of master and slave becomes blurred. The control structural diagram of the system is shown in Figure 1.

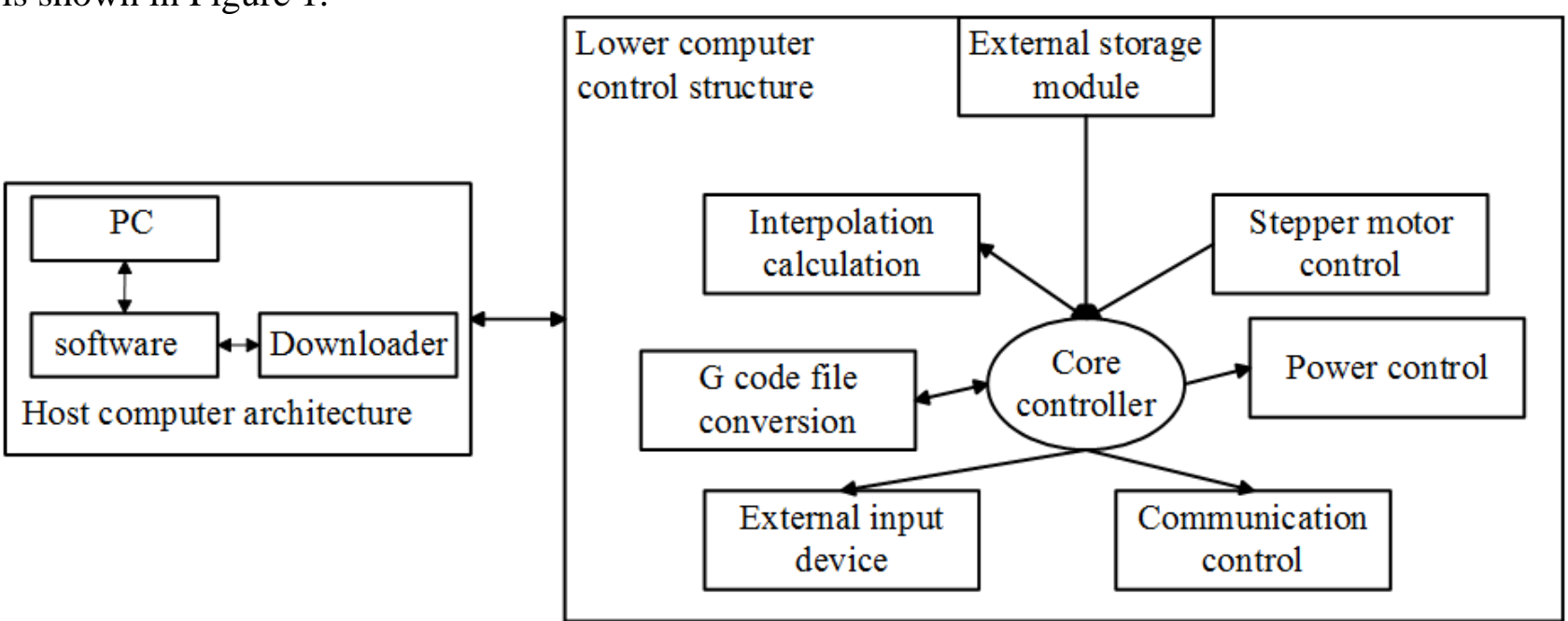

Figure. 1 The control structural diagram of the system

The Design of Mechanical Structure. The laser engraving machine generally adopts the mechanical structure similar to that of the milling planer, but differs in the relative motion mode of the fixation of working table or that of laser head. The fixation of working table means that the position of work piece remains the same, the way that the X-Y axis motor drives the laser head causes processing to be carried out; The fixation of the laser head means that the laser head is at top of the work piece and keeps invariant, and the work peace moves with the X-Y axis platform. Taking account of convenience of the design, two motors are designed to drive respectively the laser head and the working platform for motion control [4]. The system structure is shown in Figure 2.

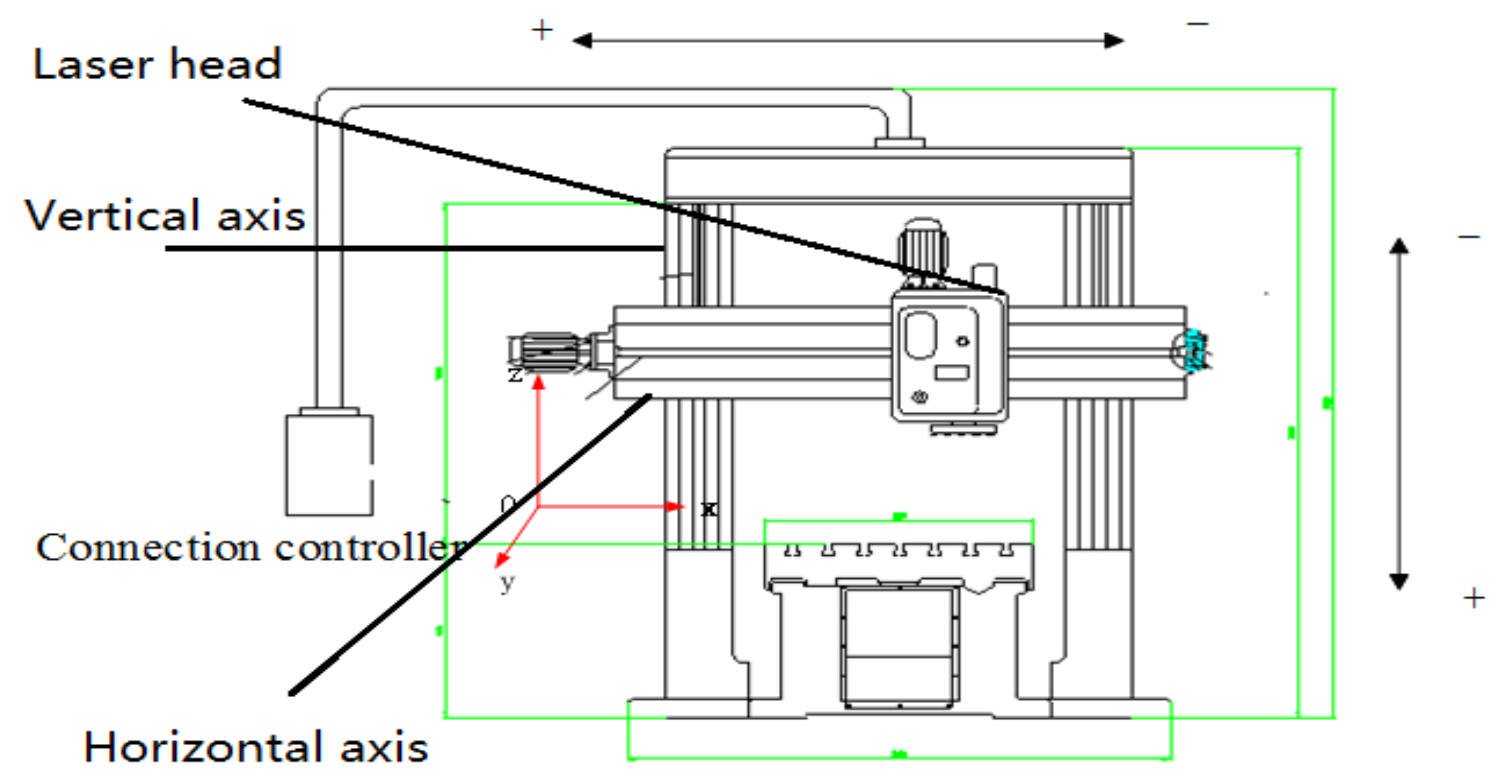

Figure. 2 Schematic diagram of mechanical structure 
Design of Interpolation Algorithm. Interpolation algorithm is an important part of the system, and the interpolation algorithm directly affects the machining accuracy. Shape outlines of parts consist of various linear (such as straight line, arc, spiral line, parabola, free curve, etc.). Therefore, controlling the movement of the cutting tool or work piece to make the processed parts meet fully the requirements of precision of geometry dimension and surface roughness is the core issue of the numerical control system (CNC system).In actual production, short line or arc is mostly used to approach the outline curve of parts, in some circumstances, parabola, ellipse and hyperbola are used to approach it. There are two kinds of interpolation algorithm: the data sampling interpolation and the reference pulse interpolation, but the program implementation of the data sampling interpolation is more difficult than that of the reference pulse interpolation with a complex operation. Because the computing power of single-chip microcomputer is limited and the stepper motor is designed to act as the opened-loop system of the driving device, the point-by-point relative method under the interpolation classification for reference pulse in Scheme 1 is adopted [5].

\section{Mechanical Modeling of the System}

Once the structural dimension of screw stepper motor is determined, the design of the rake construction can be carried out. Based on the principle of low cost, easy processing and rapid installation, by the means of fitting-up of garment parts, an acrylic plate can be used to process parts of each part, then screws can be used to fix them. In order to view the design results intuitively as well as check the bad parameters which may exist in match tolerances, SolidWorks 2013 software is used to conduct a three-dimensional modeling, and the virtual assembly of each component is completed. The assembly drawing is shown in Figure 3.

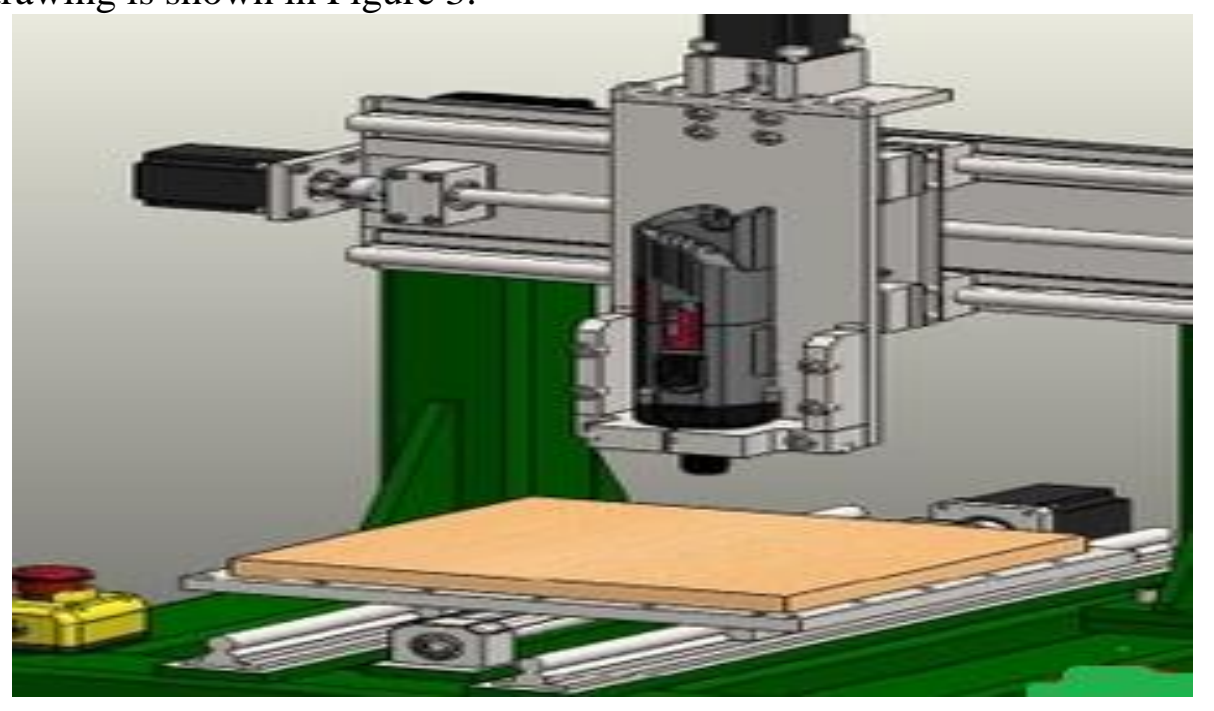

Figure. 3 The integral assembly drawing

\section{The Hardware Design of the System}

Design of Power Supply for the System. Three parts in the whole system need to be powered by power supply, namely: System of single-chip microcomputer, the driver of stepper motor and laser-power supply circuit [6]. The total power supply uses a switch power supply of 220VAC-12VDC with maximum 2A output. The system of single-chip microcomputer and the laser-power supply circuit need the power supply of $5 \mathrm{~V}$ and $<=500 \mathrm{~mA}$, so the two LM7805 chips are used respectively to carry out the two-level voltage regulation. Thus, the electromagnetic interference among each part is reduced. The driver of stepper motor supports the input of maximum 48V DC voltage, but to support various kinds of motor specifications, a LDO regulation 
chip with adjustable output voltage is used. In this system, the power supply system is very important, is directly related to the processing quality, in this system uses the $5 \mathrm{~V}$ and the $3.3 \mathrm{~V}$ voltage supply. This is because the power supply voltage control board I choose is $3.3 \mathrm{~V}$, and we know that the market regulator chip typically only $12 \mathrm{~V}, 5 \mathrm{~V}, 3.3 \mathrm{~V}$, so we should adopt special controller voltage low dropout voltage regulator chip, LP2985 as the task execution, through accurate circuit design, we passed the actual inspection, in a certain range of error, the circuit can output voltage stability 3.3V [7]. In order to stabilizee the power supply of the part, the mature voltage regulator module based on the chip on the market can be used directly, and the integrated circuit diagram of power supply module in the system is shown in Figure 4.

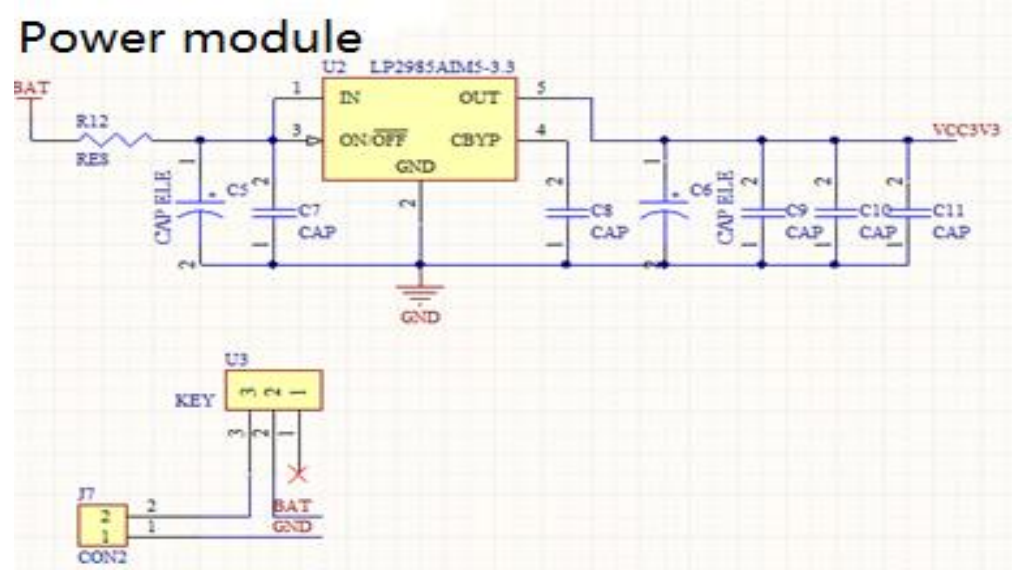

Figure.4 The schematic diagram of integrated power-supply module in the system

The Circuit Design of SD Card. The topic of the design of a small laser engraving machine to support offline work, you can identify the SD in the open file, the processing operation; the file type must be parsed G file. The reason why SD card is selected as storage medium for off-line processing is that the communication protocol of SD card is simpler than USB protocol and that communication can be carried out through the direct connection of SD card and the single-clip microcomputer IO. At present, the system of single chip microcomputer which uses USB to act as a storage medium usually adopts the corresponding conversion chip, increasing the cost of hardware. STM32F103 series chips do not support USB-OTG function, besides; the focus of the design is not the storage and reading issues of processing files. On the other hand, all laptops have card reader interfaces, and card readers of various memory cards compatible with our life are also widely used, so it is a good thing that SD card is adopted as the storage medium of processing files. Hardware SPI of STM32 shall be used to connect the corresponding pin of SD card to SPI interface of STM32 chip, $15 \mathrm{k} \Omega$ pull-up resistor is added to each line to increase the stability of communication. The schematic diagram is shown in Figure 5.

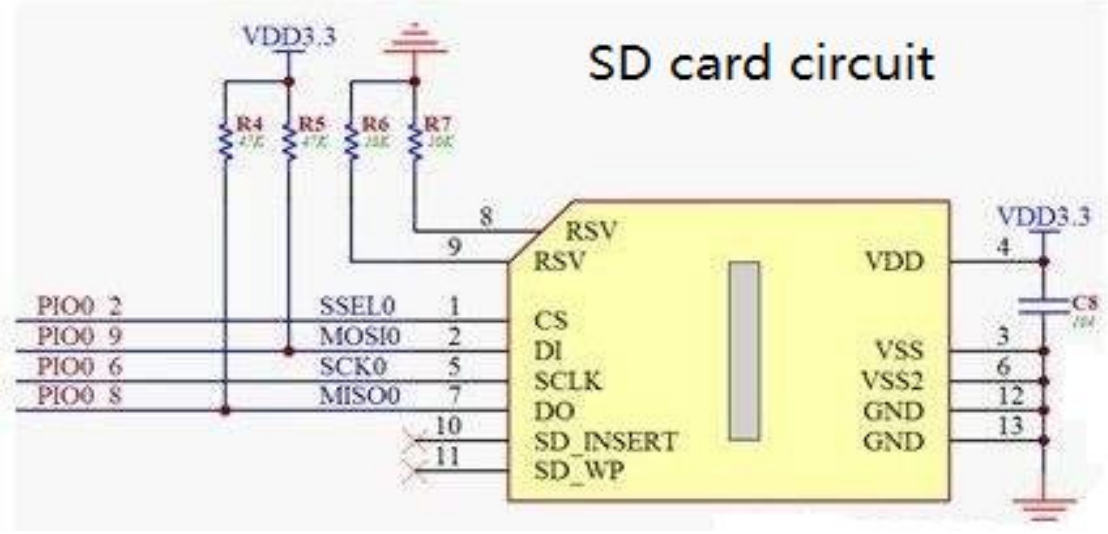

Figure. 5 The circuit of 5 SD card 
The Design of Driving Circuit for Step Motor. The driving part of stepper motor uses the driver board based on A3967SLB chip, so it is unnecessary for us to study the applied design of the chip; we only need to focus on the usage method of module. A3967 driver board is an open-source module, whose designers have released detailed information of the module on their website, based on the fundamentals of electric circuit, anyone can change and release a new version and apply it to their own design for free[8]. This module has become the first choice for many small laser engraving machines. The pin definition of the module is as shown in Figure 6.

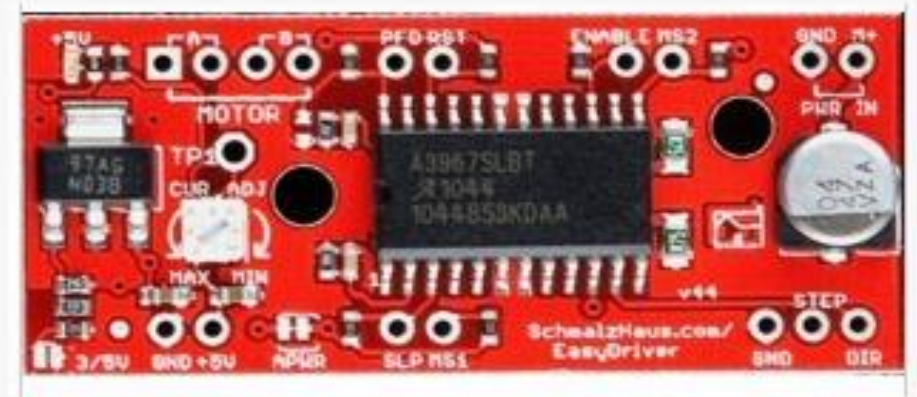

Figure. 6 The pin definition of A3967 driver board

According to the design of the project and the use of the A3967 driver board, the stepper motor drive structure schematic diagram shown in Figure 7.

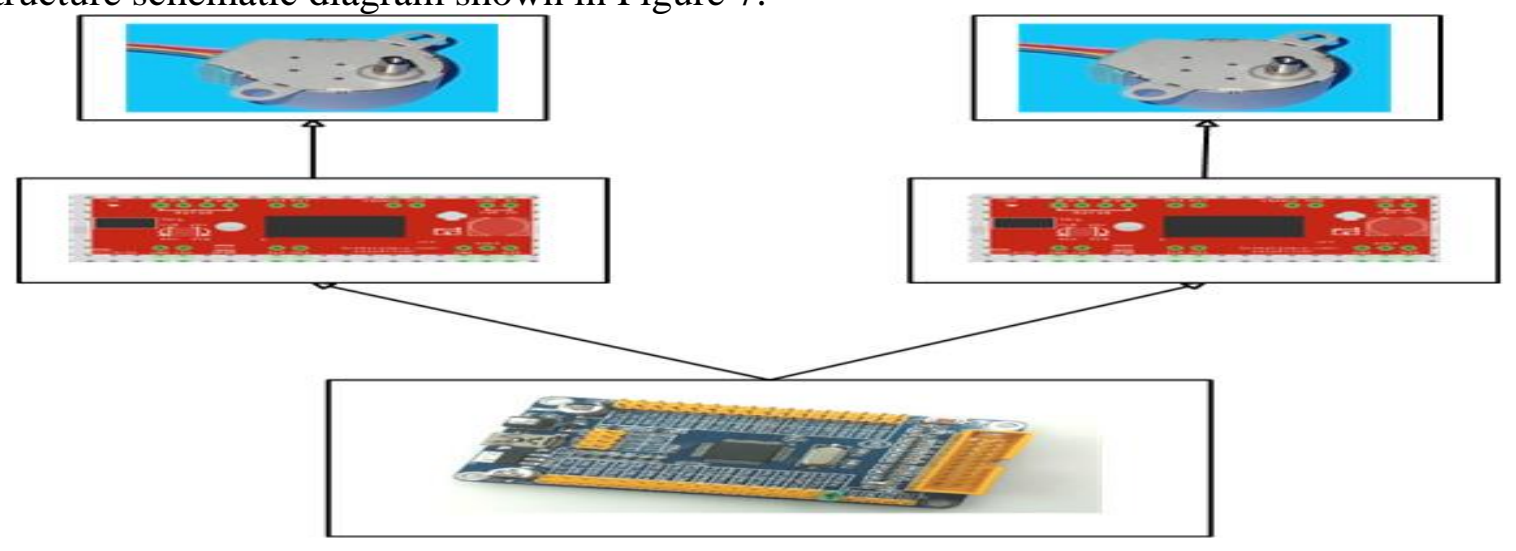

Figure.7 The drive circuit of stepper motor

\section{The Software Design of the System}

The Overall Design of the Software. Software programming plays an important role in the design of the power system, self check system, then check whether the normal communication, file system and identify whether the SD card, if any, to offline mode, call G code parser and interpolation algorithm[9], the laser head start work, for the flow charts of the software control in the system, see to Figure 8.

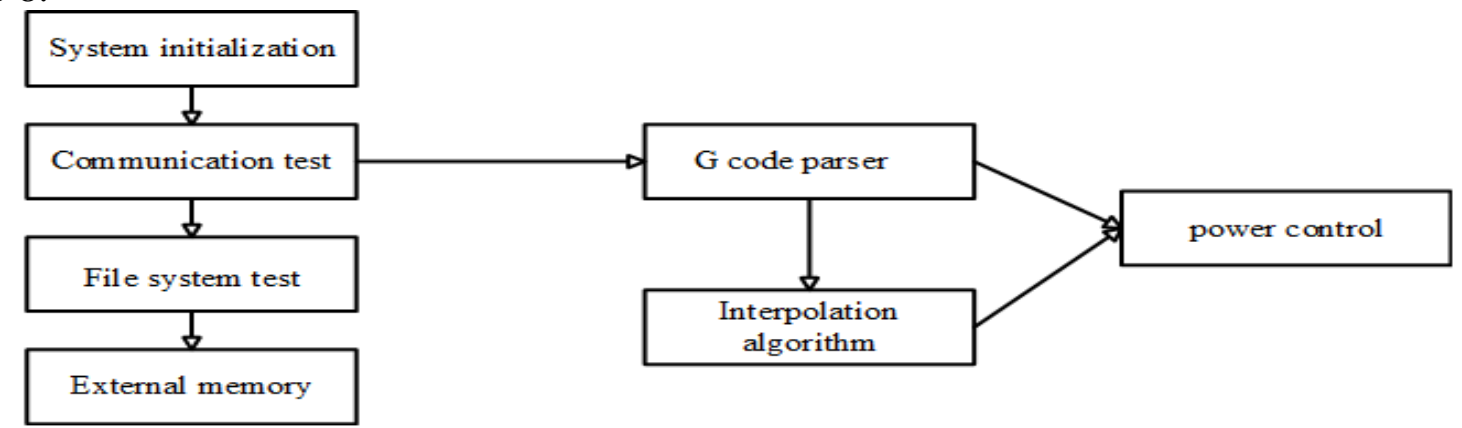

Figure. 8 The flow charts of software processing 
The Design for Driving Algorithm of the Stepper Motor. Because the driver board of the dedicated stepper motor is used, it is OK to input the pulse stepper and positive reversal signals to the driver board. This designed single-chip microcomputer IO is used to conduct the reverse of high and low levels to achieve the output of pulse signal, the maximum output frequency of IO of STM32 chip can reach $45 \mathrm{MHZ}$, meeting fully the service requirements[10].

\section{Summary}

From the aspects of mechanical design, hardware circuit design, software flow design and so on, this paper presents the realization process of the small laser engraving machine, focus on the design of the $\mathrm{G}$ code parser and the linear interpolation algorithm, through practical tests, small laser engraving machine design precision can reach $0.01 \mathrm{~mm}$, the maximum engraving area of up to $80 * 80 \mathrm{~mm}$, can achieve a good effect in the carving of cardboard, cork boards, leather and other objects, the work is stable, high reliability.

\section{References}

[1] Zhang Ke, Guo Dong, Qin Wenpu. Design of miniaturized servo controller based on STM32 [J]. Small and Special Electrical Machines, 2016, 44(5):83-86.

[2] Niu Yanmin, Su Junhong. A programmable laser energy attenuator based on STM32 [J]. Laser Technology, 2016, 40(4):592-595.

[3] Wang Chenhui, Wu Yue, Yang Kai. Design of multi-channel data acquisition system based on STM32 [J]. Application of electronic technology, 2016, 42(1):51-53.

[4] Li Hui, Xu Xiaochuan, Yu Xuewei. Design of intelligent generator electromechanical parameter measurement device based on STM32 [J]. Electronic measurement and instrument, 2016, 53(2):125-128.

[5] Yang Zhengming, Xu Zhiqun. Design of fishing oil consumption monitoring system based on STM32 [J]. Marine Engineering, 2016, 45(4):122-125.

[6] Ren Shunhang, Lv Pengpeng, Wang Lipeng. Design and implementation of STM32 based expert system [J]. Application of electronic technology, 2014, 40(1):75-78.

[7] Li Cong, Li Chunmei, ZhouZhiqun. Design and implementation of data transfer interface device based on STM32 [J]. Computer engineering and design, 2014(10):3416-3421.

[8] Li Na, Niu Xiaofei, Xu Haifeng. Smart home wireless laser warning system based on STM32 [J]. Laser Journal, 2013(5):60-62.

[9] Ma Mei, Xu Yanfei, Wang Peng. Design of intelligent power saving and non-hidden socket based on STM32 [J]. Television technology, 2014, 38(23):68-71.

[10] Chen Yingzhao, GaoYueming, Gan Zhenhua. Design of portable ECG monitor based on STM32 [J]. Application of electronic technology, 2013, 39(9):18-20. 